\title{
What explains the Great Moderation in the US? A structural analysis
}

\author{
Fabio Canova \\ ICREA-UPF, CREI, AMeN and CEPR *
}

Final version: December 2007

\begin{abstract}
This paper investigates what has caused output and inflation volatility to fall in the US using a small scale structural model using Bayesian techniques and rolling samples. There are instabilities in the posterior of the parameters describing the private sector, the policy rule and the standard deviation of the shocks. Results are robust to the specification of the policy rule. Changes in the parameters describing the private sector are the largest, but those of the policy rule and the covariance matrix of the shocks explain the changes most.
\end{abstract}

JEL classification no: E52, E47, C53

Key words: New Keynesian model, Bayesian methods, Monetary policy, Great Moderation.

${ }^{*}$ I would like to thank two editors of the journal, two anonymous referees, the participants of seminars at a number of universities and central banks for comments and constructive suggestions. '. The financial support of the Spanish Ministry of Education through the grant SEJ-2004-21682-E and of CREI is gratefully acknowledged. 


\section{Introduction}

Many researchers, including Blanchard and Simon (2000), McConnell and Perez Quiroz (2000) and Stock and Watson (2002)), have documented a marked decline in the variance of real activity and the variance and the persistence of inflation in the US since the early 1980s. While some have questioned the statistical significance of the reported changes (see Canova and Gambetti (2004) or Pivetta and Reis (2007)), there is agreement among macroeconomists that the nature and the causes of these changes should be careful investigated.

Taylor (1998), Sargent (1999), Clarida, et. al. (2000) and Lubik and Schorfheide (2004) among others, have attributed the fall to a permanent alteration in the weight that inflation receives in the objective function of the monetary authority. The popular version of the story runs as follows: the run-up of inflation in the 1970s occurred because the authorities believed that there was an exploitable trade-off between inflation and output. Since output was low following the two oil shocks, the temptation to inflate to bring output back, or above its potential level, was strong. Between keeping inflation low (and output low) or inflation high (and output high), the monetary authorities systematically choose the latter option. Hence, inflation in the long run turned out to be higher while output simply settled to its potential level. Since the 1980s, the perception of the output-inflation trade-off has changed. The Fed has learned that it was not exploitable and concentrated on the objective of fighting inflation. A low inflation regime ensued, and the predictability of monetary policy contributed to make the macroeconomic environment less volatile and the swings in inflation and output more unpredictable.

While prevalent, this view is not fully shared in the profession. Some researchers claim that monetary policy has not displayed any permanent switch; that the same policy rule characterizes most of the post WWII experience; that monetary policy has little influence on output; and that good luck is responsible for the changes (see e.g. Bernanke and Mihov (1998), Leeper and Zha (2003), Hanson (2006), Sims and Zha (2006)). Others have suggested "real" reasons to explain the volatility fall (see e.g. Ireland (1999), McConnell and Perez Quiroz (2000), Gordon (2005), or Campbell and Herkowitz (2006)). 
Some progress has been made in the investigation of these issues using empirical models where coefficients are allowed to vary over time. Sargent and Cogley (2001) and (2005), who used a reduced form time varying coefficient VAR, find evidence that supports the causation story running from monetary policy changes to changes in the rest of the economy. Canova and Gambetti (2004), Primiceri (2005), Sims and Zha (2006), who estimate structural time varying coefficients VARs, find little posterior support for this hypothesis. Since structural VARs only use a minimal amount of the restrictions implied by the current generation of DSGE models, one may wonder how truly structural the estimated relationships are. For example, Ireland (2001), Lubik and Schorfheide (2004) and Boivin and Giannoni (2006), who explicitly condition their analyses on a small scale DSGE model, do find evidence of policy instability.

This paper provides new evidence on the causes of output and inflation volatility changes by recursively estimating a small scale DSGE model with Bayesian techniques. Recursive estimation provides a short cut to more complicated analyses that allow for varying taste, technology and policy parameters into a structural model but requires estimation of second order approximations to the solution and much more time consuming posterior simulators (see Fernandez Villaverde and Rubio Ramirez (2007)). Also, relative to analyses where subsamples are arbitrarily chosen, a recursive approach allows us to obtain a more solid evidence on the nature of the time variations. Since the volatility of output (inflation) displays a U (inverted U) shaped pattern, conclusions may crucially depend on the selected break point. Bayesian methods have inferential and computational advantages over traditional maximum likelihood techniques when dealing with models which are a "false" description of the data generating process. This is important since, despite recent attempts to make them more realistic, DSGEs are still highly stylized; important relationships are modeled with black-box frictions; and ad-hoc shocks are used to dynamically span the probabilistic space of the data. In these situations, unrestricted maximum likelihood estimates are often unreasonable and asymptotic standard errors, constructed assuming that the model is "true" under the null, are uninterpretable. Posterior estimates are meaningful even for models displaying such features. A Bayesian framework is also preferable to an approach 
that obtains estimates of the structural parameters matching a subset of impulse responses in two respects: all the information of the model is efficiently used; the trade-off between identifiability and nonlinearities is dealt with in a more transparent way (see e.g. Canova and Sala (2006)).

Rather than searching for the "best" empirical model, we take a standard specification, popular in the theoretical literature, and show what it tells us about the causes of the changes experienced in the US. We consider first standard subsample analysis and then estimate the model a number times using overlapping samples, spanning a twenty year window over the period 1955-2002, and analyze the evolution of the posterior distributions of the structural parameters. Our analysis is geared to shed light on two issues. First, we would like to know which parameters are drifting over time, if any. Second, we would like to know which variation has contributed most to the observed changes in the volatility of output and inflation.

While it is common to examine this latter question via counterfactuals where parameters from different subsamples are switched (see e.g. Boivin and Giannoni (2006)), this practice violates a basic principle underlying the Lucas critique - agents are unaware that changes may repeatedly occur - and therefore fails to provide a reliable answer. Our approach will be to estimate unrestricted and restricted specifications, examine by how much the fit of the model changes and the consequences of restricting some parameters on fraction of output and inflation variability explained by the model.

We find instabilities in all the parameters of interest. Consistent with the common wisdom, the inflation coefficient in the policy rule increases if the sample includes only the years after 1982. However, changes are relatively small and often insignificant. The parameters describing the private sector also change and variations are significantly larger. Finally, the covariance matrix of the shocks changes over time and the adjustments are broadly in line with those reported in the VAR literature. These results are robust to the choice of policy rule: a rule which makes the interest rate responds to output growth rather than the output gap, or to future rather than current developments in the economy produce qualitatively similar results. 
We show that changes in the parameters of the policy rule and the covariance matrix of the shocks are the most important to account for the changes in the volatility of output and inflation: restricting them to be unchanged over the samples makes the fit of the model drop dramatically and the decline in volatility disappear. Interestingly, restricting the parameters of the policy rule imply a much higher inflation volatility, while restricting the standard deviations of the shocks increases the variance of output by about 10 times. Hence, the changes in the volatility of the two variables may have different causes.

In sum, it appears that both the "good policy" and the "good luck" hypotheses have some support in the data. However, it is only by combining the two that one can jointly account for the decline in the variability of real activity and inflation over time.

The rest of the paper is organized as follows. Section 2 describes the model and the estimation technique. Section 3 presents the basic results and a few robustness exercises. Section 4 compares our results to those in the literature. Section 5 studies what explains the observed changes in the volatility of output and inflation. Section 6 concludes.

\section{The model and the estimation approach}

The model we consider is a standard three equations New-Keynesian model, composed of a log-linearized Euler equation, a forward looking Phillips curve and a monetary policy rule. The system in log-linear form is:

$$
\begin{aligned}
x_{t} & =E_{t}\left(x_{t+1}\right)-\frac{1}{\varphi}\left(i_{t}-E_{t} \pi_{t+1}\right)+e_{1 t} \\
\pi_{t} & =\beta E_{t} \pi_{t+1}+\kappa x_{t}+e_{2 t} \\
i_{t} & =\psi_{r} i_{t-1}+\left(1-\psi_{r}\right)\left(\psi_{\pi} \pi_{t}+\psi_{x} x_{t}\right)+e_{3 t}
\end{aligned}
$$

where $\beta$ is the discount factor, $\phi$ is the relative risk aversion coefficient, $\kappa$ is a parameter regulating the slope of Phillips curve while $\left(\psi_{r}, \psi_{\pi}, \psi_{x}\right)$ are policy parameters. Here $x_{t}$ is the output gap, $\pi_{t}$ the inflation rate and $i_{t}$ the nominal interest rate. The shocks attached to each equation may not be structural in the sense that they may represent linear combinations 
of primitive disturbances to the economy. We assume

$$
\begin{aligned}
e_{1 t} & =\rho_{1} e_{1 t-1}+v_{1 t} \\
e_{2 t} & =a_{12} e_{1 t}+\rho_{2} e_{2 t-1}+v_{2 t} \\
e_{3 t} & =a_{13} e_{1 t}+v_{3 t}
\end{aligned}
$$

where $\rho_{1}, \rho_{2}$ capture the persistence of the shocks, $a_{12}, a_{13}$ the cross equation effects while $v_{j t}$ are mean zero processes with variance $\sigma_{j}^{2}, j=1,2,3$.

A system of equations like (1)-(3) can be obtained from a standard dynamics stochastic general equilibrium model with sticky prices, monopolistic competition and preferences which are additive in consumption and leisure when labor is the only productive factor (see e.g. Clarida, et. al. (1999)). The specification of the policy rule is consistent with the idea that the monetary authority observes current values of the output gap and of inflation when deciding the current interest rate and that policy changes are smooth, in the sense that interest rate movements may be persistent. The specification for the error terms reflects the fact that the expected level of potential output is omitted from the estimated specification and the monetary authorities may pay attention to potential output changes when taking their decisions (see also An and Schorfheide (2007)); the $\operatorname{AR}(1)$ assumption on $e_{1 t}$ and $e_{2 t}$, on the other hand, is quite standard.

Throughout this paper we use a statistically computed measure of the output gap rather than the deviation of output from the level obtained in the flexible price equilibrium. We chose this approach for two reasons. First, this choice ensures comparability with previous work. Second, a flexible price measure which does not take into account capital accumulation is likely to be misspecified and this may potentially distort inference.

Several authors, including Smets and Wouters (2003), Rabanal and Rubio Ramirez (2005) and others, have specified more complicated and realistic structures, which allow for additional shocks and frictions. Rather than add bells and whistles to the specification to generate a model with a good fit, we perform our exercises with a simple and internally consistent specification, close to those used in theoretical discussions,

The model contains 13 parameters: 6 which have some structural interpretation $\alpha_{1}=$ 
$\left(\beta, \varphi, \kappa, \psi_{r}, \psi_{x}, \psi_{\pi}\right)$ and 7 auxiliary ones, $\alpha_{2}=\left(\rho_{1}, \rho_{2}, a_{12}, a_{13}, \sigma_{1}^{2}, \sigma_{2}^{2}, \sigma_{3}^{2}\right)$. Our exercise is geared to obtain posterior distributions for $\alpha_{T}=\left(\alpha_{1 T}, \alpha_{2 T}\right)$ over different samples $T$ and to compare the time series properties of their posterior distributions. Our system can be solved using standard first order log-linear methods. The solution has a state space format

$$
\begin{aligned}
y_{1 t+1} & =A_{1}(\alpha) y_{1 t}+A_{2}(\alpha) v_{t+1} \\
y_{2 t} & =A_{3}(\alpha) y_{1 t}
\end{aligned}
$$

where $y_{2 t}=\left[\pi_{t}, x_{t}, i_{t}\right], y_{1 t}=\left[\pi_{t-1}, x_{t-1}, i_{t-1}, e_{1 t}, e_{2 t}, e_{3 t}\right]$ and the matrices $A_{i}(\alpha), i=1,2,3$ are nonlinear functions of the structural parameters $\alpha$.

Bayesian estimation of (8) is simple: given some $\alpha$, we compute the likelihood of the model, denoted by $f\left(y_{T} \mid \alpha\right)$, by means of the Kalman filter and the prediction error decomposition. Then, for any specification of the prior distribution, denoted by $g(\alpha)$, the posterior distribution for the parameters is $g\left(\alpha \mid y_{T}\right)=\frac{g(\alpha) f\left(y_{T} \mid \alpha\right)}{f(y)}$. The analytical computation of the posterior is impossible in our setup since the denominator of the expression, $f(y)$, can be obtained only by integrating $g(\alpha) f\left(y_{T} \mid \alpha\right)$ with respect to $\alpha$, a 13 dimensional vector. To obtain numerically a sequence from this unknown posterior, we employ a Metropolis algorithm. Roughly speaking, given $\alpha_{0}$ and a transition function satisfying regularity conditions, we can produce a sequence from the unknown posterior, iterating on this transition function, after discarding an initial set of draws. We choose a standard random walk transition with jumps which are taken from a normal distribution centered at zero and covariance matrix equal to a scaled version of the Hessian at the mode. The scale is sample dependent and chosen to ensure that an appropriate number of draws is accepted (between 20-50 percent). For each sample we draw 5 chains of 50000 elements each and check convergence using standard CUMSUM methods. Posterior distributions are constructed using the last 5000 draws from each of the chains.

We assume that the prior distribution can be factored as $g(\alpha)=\prod_{i=1}^{13} g\left(\alpha_{i}\right)$. Prior distributions are selected according to the following rule: gamma distributions are used for parameters which must be positive; beta distributions for parameters which must lie in an interval; normal distributions for all other parameters. This implies that $\varphi, \kappa$ and $\sigma_{j}^{2}, j=$ 
$1,2,3$ have gamma priors, $\beta, \psi_{r}, \rho_{1}, \rho_{2}$ have beta priors and that the other parameters have normal priors, except for $\psi_{\pi}$, whose prior is truncated below 1.0. The mean and the standard deviation of these distributions are in table 1.

Clarida et. al. (2000) and Lubik and Schorfheide (2004) among others, have emphasized the potential importance of indeterminacies to characterize the US experience over the last 35 years. Since our prior distribution for the inflation coefficient in the policy rule is truncated at one, no indeterminacy is allowed. Therefore, the changes we emphasize are changes within a determinate regime rather than changes across regimes. Canova and Gambetti (2007) showed that the dynamics induced by this model under indeterminacy (continuity solution) can be reasonably matched in a system where only determinate equilibria are considered. Hence, considering only determinate equilibria is less restrictive than it may originally appear. Also, since our samples cut across periods with potentially different regimes, our prior assumption that the policy coefficient on inflation is larger or equal to one on average is not inconsistent with the possibility that in particular periods of the sample such a restriction is not satisfied.

The means of the priors are located around standard calibrated values - the one for $\kappa$ reflects a-priori knowledge about its underlying components. The selected standard deviations imply proper but non-informative densities over a range of economically reasonable parameter values. We select "loose" priors to minimize subjective information and to allow the posterior to move away from the prior if the data is informative. Since we maintain the same prior in every subsample, differences in the location and in the shape of the posterior will tell us how much the likelihood evolves over time.

The data is quarterly for the sample 1955:1-2002:1 and it is the same as in Ireland (2004). The output gap is proxied by GDP in deviation from a linear trend, inflation is measured as quarter-on-quarter log changes in CPI, and the nominal interest rate we use is the Federal funds rate. Since output is linearly detrended once and for the whole sample, trend breaks can not explain the changes we are interested in.

We estimate the model over a number of samples. We start from the [1955:1, 1974:4] sample and repeat estimation moving the starting and ending date by four years, so as to 
keep the size of the window constant to 20 years. Keeping a fixed window size is important in order to minimize differences produced by different precision of the estimates. The last subsample is [1983:1-2002:1], which means that we produce 8 posterior distributions for the parameters.

\section{Results}

Before we describe the estimation results, we plot in figure 1 the variance of the three variables in percentage terms in various samples. This plot may help to better understand the reasons of our study and the estimates we obtain.

Three features of figure 1 are important. First, there is a fall in the variance of inflation only if the sample starts in, at least, 1982. Samples which include any year preceding 1982 display a variance which is much higher and roughly unchanged. Second, the variance of the output gap is U-shaped, with the flex point represented by the 1967-1986 sample. This means that, for appropriately selected samples, one can claim that the variability of the output gap has fallen or risen over time (compare, for example, the 1959-1978 and 1983-2002 samples with 1963-1982 and 1983-2002 samples). In general, the absence of a once-and-for-all break makes the rolling analysis more informative than subsample exercises when studying the reasons for the changes. Third, the variance of the nominal interest rate shows a inverted U-shaped pattern. Interesting, the pre-1979 and post-1982 volatilities are almost identical while any sample which includes part or all of the Volker experiment, produces a much higher volatility of the nominal interest rate. Once again, our rolling analysis may shed some light for why this pattern may emerge. 


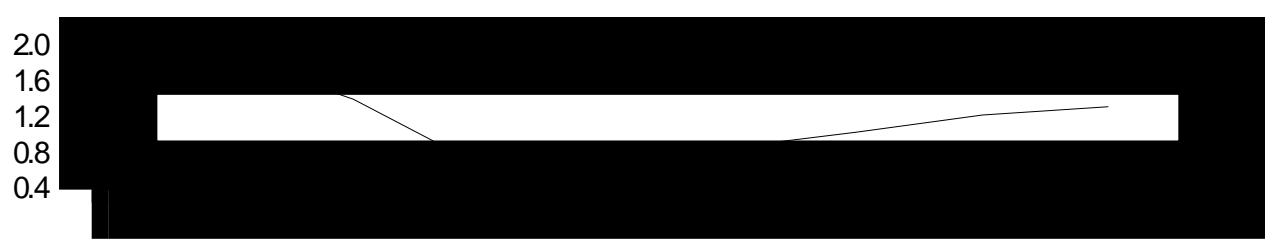

Output gap

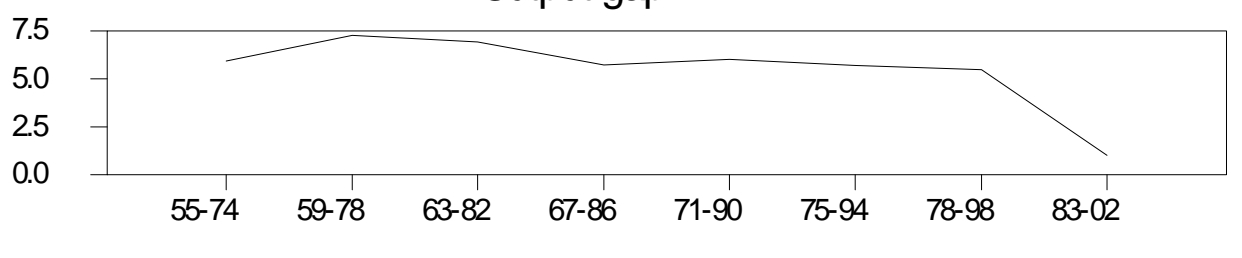

Inflation

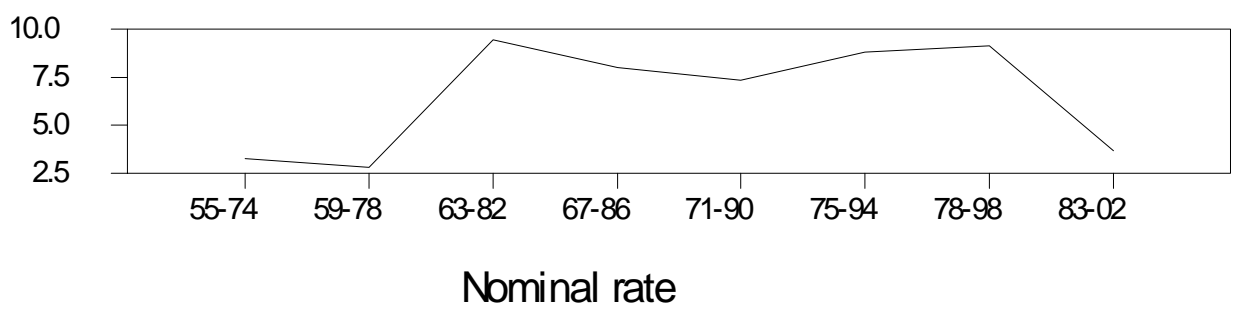

Figure 1: Variances, different samples

\subsection{Evidence for subsample estimation}

We start by presenting results for the 1955:1-2002:1 sample and for three subsamples commonly employed in the literature (1955:1-1979:2, 1979:3-2002:1, 1982:4-2002:1). We are interested in two issues: we want to assess in which dimension the structural system changes to cope with the time profile of the volatility of output and inflation documented in figure 1; and to see how distorted inference is when potential heterogeneities in the process generating the data are not accounted for.

Table 1 presents the posterior mean and the highest 95 percent posterior interval (HPI) for each of the parameters in each sample. This measure, which corresponds to classical confidence intervals, tells us where 95 percent of the mass of the posterior distribution is located. For distributions which are skewed or multimodal, the interval need not contain the posterior mean, which is precisely the case for certain subsamples, or could have disjoint pieces. 


\begin{tabular}{|c|c|c|c|c|c|c|c|c|c|}
\hline & \multirow{3}{*}{\begin{tabular}{|c} 
Prior \\
Mean Std
\end{tabular}} & \multicolumn{8}{|c|}{ Posterior } \\
\hline & & \multicolumn{2}{|c|}{ 1955-2002 } & \multicolumn{2}{|c|}{ 1955-1979 } & \multicolumn{2}{|c|}{ 1979-2002 } & \multicolumn{2}{|c|}{ 1982-2002 } \\
\hline & & Mean & HPI & Mean & HPI & Mean & HPI & Mean & HPI \\
\hline$\varphi$ & $2.0 \quad 0.35$ & 0.47 & {$[0.42,0.52]$} & 3.04 & {$[2.67,3.51]$} & 0.87 & {$[0.25,0.41]$} & 0.74 & {$[0.45,1.01]$} \\
\hline$\beta$ & $\begin{array}{ll}0.98 & 0.01\end{array}$ & 0.98 & {$[0.98,0.99]$} & 0.979 & {$[0.97,0.99]$} & 0.97 & {$[0.95,0.99]$} & 0.97 & {$[0.95,0.99]$} \\
\hline$\kappa$ & $2.0 \quad 1.0$ & 4.15 & {$[4.10,4.22]$} & 3.42 & {$[3.07,3.93]$} & 3.90 & {$[3.84,4.22]$} & 3.90 & {$[3.42,4.22]$} \\
\hline$\psi_{r}$ & $0.8 \quad 0.25$ & 0.98 & {$[0.98,0.99]$} & 0.99 & {$[0.99,0.99]$} & 0.98 & {$[0.98,0.99]$} & 0.98 & {$[0.98,0.99]$} \\
\hline$\psi_{x}$ & $0.5 \quad 0.25$ & 0.02 & {$[0.00,0.08]$} & 0.16 & {$[0.01,0.50]$} & 0.03 & {$[-0.00,0.01]$} & 0.01 & {$[0.00,0.02]$} \\
\hline$\psi_{\pi}$ & $1.3 \quad 0.5$ & 1.71 & {$[1.65,1.76]$} & 1.44 & {$[1.42,1.50]$} & 1.54 & {$[1.23,1.53]$} & 1.57 & {$[1.51,1.58]$} \\
\hline$\rho_{1}$ & 0.850 .25 & 0.97 & {$[0.97,0.98]$} & 0.94 & {$[0.92,0.94]$} & 0.97 & {$[0.97,0.97]$} & 0.95 & {$[0.94,0.96]$} \\
\hline$\rho_{2}$ & $\begin{array}{lll}0.85 & 0.25\end{array}$ & 0.99 & {$[0.99,0.99]$} & 0.99 & {$[0.99,0.99]$} & 0.99 & {$[0.99,0.99]$} & 0.99 & {$[0.99,0.99]$} \\
\hline$\sigma_{1}$ & 0.010 .50 & 0.012 & {$[0.011,0.012]$} & 0.021 & {$[0.016,0.029]$} & 0.012 & {$[0.012,0.012]$} & 0.012 & {$[0.012,0.012]$} \\
\hline$\sigma_{2}$ & $\begin{array}{lll}0.01 & 0.50\end{array}$ & 0.079 & {$[0.071,0.081]$} & 0.047 & {$[0.044,0.061]$} & 0.071 & {$[0.067,0.087]$} & 0.076 & {$[0.065,0.078$} \\
\hline$\sigma_{3}$ & $0.01 \quad 0.50$ & 0.156 & {$[0.156,0.167]$} & 0.031 & {$[0.012,0.048]$} & 0.131 & {$[0.132,0.203]$} & 0.146 & {$[0.117,0.160]$} \\
\hline$a_{12}$ & $0.0 \quad 0.25$ & -0.33 & {$[-0.66,-0.31]$} & 0.01 & {$[-0.48,0.72]$} & -0.00 & {$[-0.40,0.32]$} & -0.01 & {$[-0.46,0.23]$} \\
\hline$a_{13}$ & $\begin{array}{ll}0.0 & 0.25\end{array}$ & 0.15 & {$[0.27,0.53]$} & 0.52 & {$[0.10,0.87]$} & 0.02 & {$[-0.22,0.20]$} & -0.10 & {$[-0.51,0.31]$} \\
\hline $\begin{array}{l}\text { Acceptance rate } \\
\text { Convergence after }\end{array}$ & & $\begin{array}{c}0.28 \\
25000\end{array}$ & draws & $\begin{array}{c}0.55 \\
29000\end{array}$ & draws & $\begin{array}{c}0.21 \\
30000\end{array}$ & draws & $\begin{array}{c}0.38 \\
29000\end{array}$ & draws \\
\hline
\end{tabular}

Table 1: Prior and Posterior Moments, Basic rule

There are several interesting aspects of table 1 we would like to emphasize. First, the samples are informative for all parameters of interest. In fact, the location changes and the spreads of the posteriors are smaller than those of the prior. Therefore, the identification problems Canova and Sala (2006) have highlighted in the context of this model, appear to be less dramatic with the selected parameterization. The mean estimate of $\kappa$ is typically larger than the estimates available in the literature (which are of the order of 0.5). We can obtain mean estimates of $\kappa$ in that range if estimation is performed conditional on $a_{12}=0$. Hence, one can conjecture that either misspecification or the impossibility to separate $\kappa$ and $a_{12}$ is responsible for the differences.

Second, splitting the sample in two changes the point estimates of the policy parameters, with full sample estimates been closer to the 1982-2002 estimates. Cross sample variations in $\psi_{x}$ and $\psi_{r}$ are small or insignificant. However, consistent with the conventional wisdom the second subsamples are characterized by a higher $\psi_{\pi}$ and the differences, at least for the latest subsample, are statistically significant - HPIs for the 1955-1979 and 1982-2002 samples do not overlap.

Third, two of the parameters characterizing the private sector, the risk aversion coeffi- 
cient $\varphi$ and the Phillips curve trade-off $\kappa$, display considerable changes. The point estimate of $\varphi$ dramatically drops in the last two subsamples and the HPIs do not overlap with the one of the first sample; the point estimate of $\kappa$ increases, but the uncertainty around the point estimate is sufficiently large to make changes a-posteriori insignificant.

Fourth, using as the second subsample 1979-2002 or 1982-2002 makes little difference for the point estimates we obtain. However HPIs do change: excluding the 1979-1982 period makes the posterior intervals for $\psi_{\pi}, \sigma_{2}, \sigma_{3}$ smaller and those of $\varphi$ and $\kappa$ larger. Since excluding the Volker experiment from the sample makes information on the location of the private sector parameters weaker, one must conclude that it is information present in this period that identifies the location of the posterior distribution of these parameters.

Fifth, the covariance matrix of the shocks displays considerable changes. The standard deviation of the shocks to the Euler equation is larger in the first subsample, while the standard deviations of the shocks to the other two equations are larger after 1979. Excluding the 1979-1982 period does not change the point estimates of the standard deviations but their HPIs are centered around a lower value if estimation starts at 1982 . The covariance terms have HPIs which are entirely on one side of zero for the full sample but generally not in the subsamples. Hence, the statistical correlation one finds in the full sample may be spurious.

Sixth, as table 2 shows, regardless of the sample, the model tends to underestimate the variance of the output gap, while it overestimates the variance of inflation by a substantial amount, even 10 times in some samples, when one uses posterior mean estimates to compute the variabilities implied by the model. Moreover, the actual values of output variability are in the upper tail of the estimated posterior distribution of output variability of any sample, while the actual values of the inflation variance are in the very low tail of the estimated posterior distribution of the inflation variance. In other words, the specification is too simple to be able to jointly account for the variability of the two variables. Nevertheless, the model captures the fall in variability across subsamples: when going from the 1955-1979 sample to the 1979-2002 or 1982-2002 samples the estimated variance of output drops by about 50 percent (1.61 to $0.60 / 0.80)$ and the one of inflation falls by about two-thirds (47.9 


\begin{tabular}{|c|c|c|c|c|}
\hline & 1955-2002 & $1955-1979$ & 1979-2002 & $1982-2002$ \\
\hline $\operatorname{Var}^{d}(\mathrm{y})$ & 5.76 & 2.16 & 1.79 & 1.38 \\
\hline $\operatorname{Var}^{d}(\pi)$ & 5.95 & 6.92 & 4.75 & 1.06 \\
\hline & \multicolumn{4}{|c|}{ Basic rule } \\
\hline Mean Posterior Var(y) & 3.55 & 1.61 & 0.60 & 0.80 \\
\hline Percentile where $\operatorname{Var}^{d}(\mathrm{y})$ lies & 83 & 80 & 89 & 90 \\
\hline Mean Posterior $\operatorname{Var}(\pi)$ & 74.13 & 47.90 & 12.72 & 16.39 \\
\hline \multirow[t]{2}{*}{ Percentile where $\operatorname{Var}^{d}(\pi)$ lies } & 01 & 05 & 13 & 02 \\
\hline & \multicolumn{4}{|c|}{ Output growth rule } \\
\hline Mean Posterior Var(y) & 0.20 & 0.18 & 1.25 & 1.08 \\
\hline Percentile where $\operatorname{Var}^{d}(\mathrm{y})$ lies & 100 & 99 & 80 & 82 \\
\hline Mean Posterior $\operatorname{Var}(\pi)$ & 7.50 & 3.50 & 26.92 & 31.84 \\
\hline \multirow[t]{2}{*}{ Percentile where $\operatorname{Var}^{d}(\pi)$ lies } & 34 & 97 & 03 & 02 \\
\hline & \multicolumn{4}{|c|}{ Forward rule } \\
\hline Mean Posterior Var(y) & 4.34 & 0.27 & 0.11 & 0.11 \\
\hline Percentile where $\operatorname{Var}^{d}(\mathrm{y})$ lies & 72 & 97 & 98 & 99 \\
\hline Mean Posterior $\operatorname{Var}(\pi)$ & 13.44 & 5.47 & 4.12 & 2.81 \\
\hline Percentile where $\operatorname{Var}^{d}(\pi)$ lies & 17 & 60 & 54 & 28 \\
\hline
\end{tabular}

Table 2: Data and Estimated Posterior variabilities

to $12.7 / 16.3)$.

Campbell and Herkovitz (2006) have suggested that changes in the credit constraints faced by consumers in the early 1980s could account for the fall in inflation and output volatilities observed after that date. In their model, volatility drops because labor supply (and therefore real activity) is very sensitive to shocks when credit constraints are binding and much less when constraints are relaxed. In our model labor supply decisions are absent, therefore such an effect is unmeasured. Nevertheless, changes in the risk aversion coefficient could play a similar role. In section 5 we study whether variations in the elasticity of the output gap to real interest rate changes can account for part of the volatility changes.

Arias, et. al, (2006) have argued that to account for the fall in output volatility, one need not change the parameters of the model across subsamples, but simply allow the variance of the Solow residuals to be reduced over time. While the model we use is different, making the comparison difficult, our results seem to tell a different story. Given that the parameters of the private sector have changed, the variance of the Phillips curve shock increases, rather decreases, after 1979 to fit the evidence.

The increase in the standard deviation of the shock to the interest rate equation may 
appear odd. However, one should be careful in comparing our estimates to those present in the literature, since disturbances in the model do not necessarily have a structural interpretation. We have run a VAR with the same three variables and computed the standard deviation of the reduced form residuals of the interest rate equation in the three subsamples and found a similar pattern. Hence, to fit the time path for the endogenous variables, we need a combination of changes in the parameters of the model. Given the pattern for the variance of interest rates presented in figure 1, such combination must include an increase in the volatility of the residuals of this equation.

Overall, the analysis of this section has highlighted two important conclusions. First, changes in the parameters of the policy rule do occur but their magnitude is smaller than often emphasized in the literature. Second, coefficients describing the private sector and the standard deviation of the shocks display large and significant changes. The next subsection examines whether these conclusions remain valid when posterior distributions are obtained over rolling samples with a window of 20 years.

\subsection{Evidence from rolling estimation}

We have argued that arbitrarily breaking the sample in two is less than an ideal approach for what we want to investigate. Two reasons make the results potentially difficult to interpret. First, using fixed subsamples forces all the relationships of the model to break at the same date - clearly violating what we have displayed in figure 1 - and this may induce important biases. Second, the pattern that the level and the variability of output and interest rates displays does not fit well into the null of stability nor the alternative of a permanent jump. Therefore, the conclusions one draws may be highly sensitive to the choice of break date. Our rolling estimation approach does not entirely solve these problems. To account for them we would need to estimate the model allowing structural parameters to be fully time varying. Nevertheless, by comparing posterior estimates over different samples, we can provide a more robust characterization of the changes observed over that last 35 years than simply using (fixed) subsample analysis.

We plot the posterior mean (straight line) and HPI estimates (dashed lines) obtained 
in the 8 subsamples for the parameters of interest in figure 2. The figure confirms and qualifies the conclusions we have previously reached. There are considerable variations in both the posterior mean and the posterior HPI for the coefficient of relative risk aversion $\varphi$ over samples. While variations are present in samples which include years before 1982, it is only after that date that the fall becomes considerable and significant. The Phillips curve trade-off $\kappa$ is increasing over time in a manner which is consistent with the previous analysis: the trend is clear but the posterior significance of the changes is low.
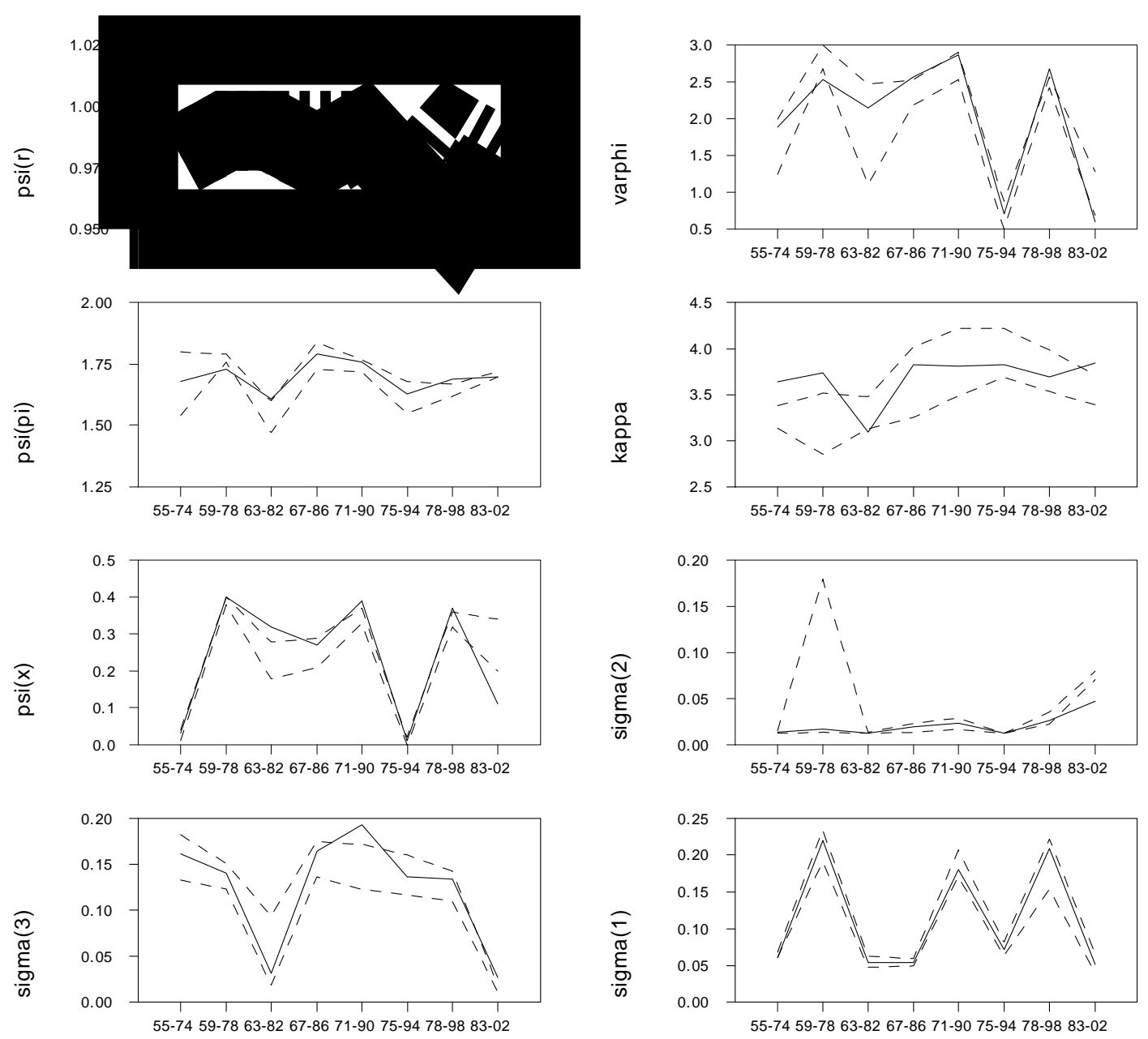

Figure 2: Posterior mean estimates and HPI, different samples

The Phillips curve trade-off in more structural versions of the model we consider is typically regulated by a (nonlinear) function of four parameters: the coefficient of relative risk 
aversion, the inverse elasticity of labor supply, the discount factor, and the price stickiness parameter. While there is little evidence that the price stickiness parameter has changed over time, at least in micro studies, and since estimates of the discount factor do not display important variations over the samples, one must conclude that variations in the intertemporal elasticity of labor supply must counteract variations in the risk aversion coefficient and give the mild trend we observe in $\kappa$. It is tempting to associate this trend with the changes that the US labor market experienced over period (higher female participation, larger number of migrant workers, etc.). However, one should realize that more general specifications of the model (for example, with decreasing returns to scale production) produce more complicated Phillips curve trade-off where other parameters enter. Rather than forcing an explanation on a model which is not designed to do this, we leave the question of what drives the trend in $\kappa$ for future research.

The parameters of the policy rule display minor variations across samples: HPIs for different samples almost always overlap, except for the coefficients on the output gap. Consistent with the analysis of Bernanke and Mihov (1998), the pattern present in figure 2 squares well with the idea that none of the three policy coefficients has permanently shifted over time. Also, consistent with Canova and Gambetti (2004), our recursive posterior analysis shows that the policy rule during Burns and Greenspan tenures were not too different. Taking for granted that the policy rule represents the actual policy well over the entire period, HPIs for the policy coefficients in the earlier and the later samples overlap.

Fourth, the standard deviation of two of the three disturbances $\left(\sigma_{2}, \sigma_{3}\right)$ display considerable variations over subsamples. Since also the covariance parameters - not displayed here - display this feature, it is the entire covariance structure of the disturbances that is significantly altered over time.

Given these results, the temptation to associate variations in the variance of the output gap over time with changes in $\varphi$ and variations in the variances of inflation and interest rates with changes in the covariance structure of the disturbances is strong. To make the link more transparent we estimate in section 5 restricted systems where certain parameters are fixed at their 1955-1974 mean value. By comparing restricted vs. unrestricted estimates 
one can obtain a formal indication of what parameters contributed most to the variations in the variance of output and inflation.

\subsection{Robustness}

The model we employ is rather standard. However, the specification of its details may be subject to some debate. In particular, while we have chosen to work with a policy rule where the nominal interest rate depends on the current output gap and current inflation, a policy rule specified in terms of current output growth and current inflation is probably equally reasonable. Furthermore, some literature (see e.g. Clarida et. al. (2000), Boivin and Giannoni (2006)) specifies a forward looking rule where the current interest rate responds to future expected changes in the output gap and in inflation. Would the main conclusions change if one of these alternative rules is used? Evidence on this issue is in tables 3 and 4, which report posterior means and HPIs for the full sample and the three subsamples presented in table 1, for the two alternative rules. Results obtained using rolling samples are comparable and therefore not presented.

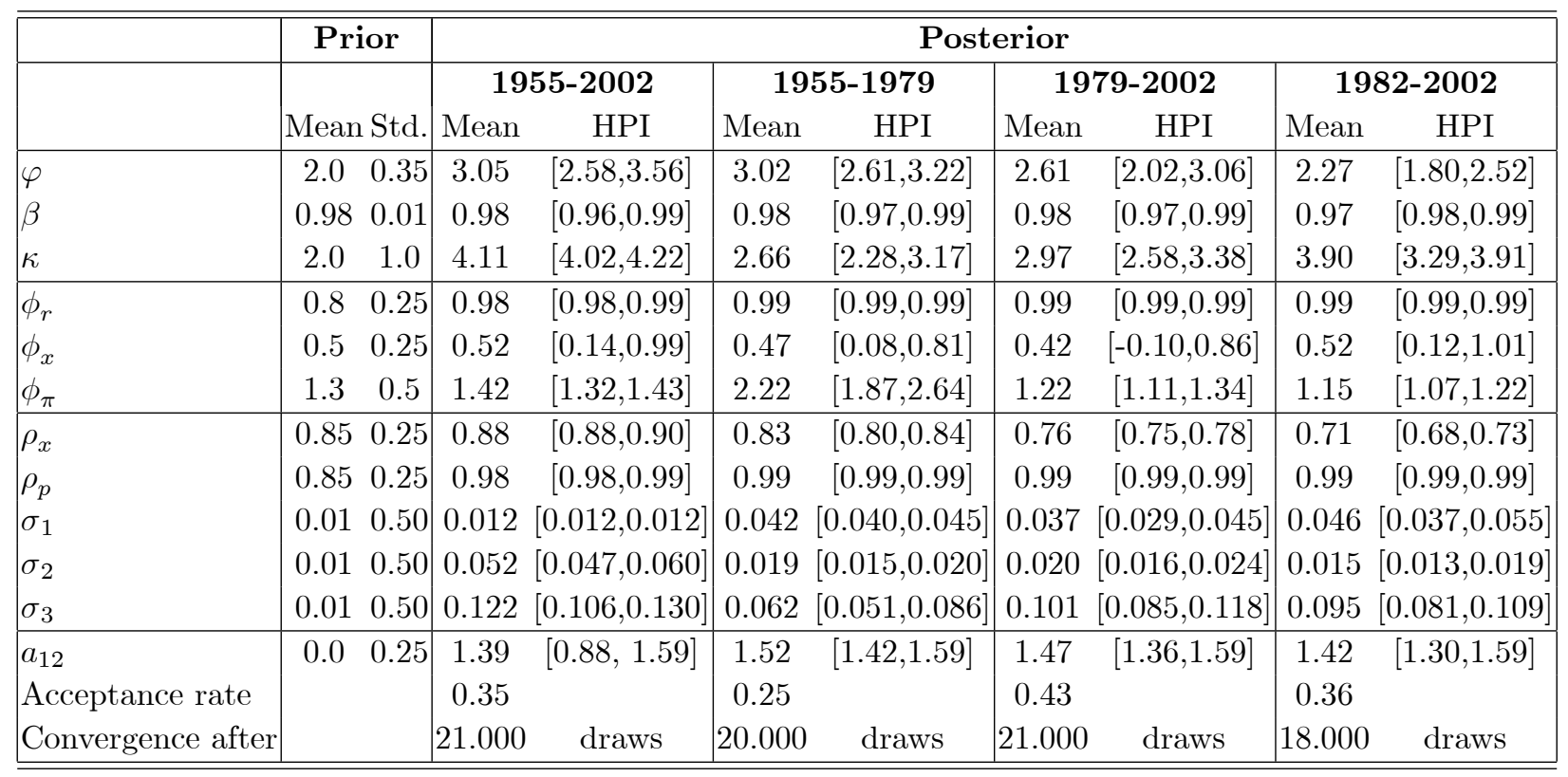

Table 3: Prior and Posterior Moments, Output growth rule

It is well known that the statistical output gap proxy we use is subject to a large amount of measurement error. Consequently, estimates of the structural parameters may 
fail to have the "right" magnitudes because a large amount of measurement error is present in each sample. We have already argued that model based measures of the output gap do not seem to be the solution as they are typically obtained disregarding the role of the capital stock. Since Orphanides (2004) has argued that measurement errors are significantly reduced if output growth is used in place of the output gap, it is worth investigating what happens to our estimates when we employ this new policy equation.

Table 3 indicates that the coefficient of relative risk aversion $\varphi$ falls but now the fall is much more limited in magnitude. Nevertheless, HPIs for the 1955-1979 and 1982-2002 samples do not overlap. Estimates of the Phillips curve trade-off $\kappa$ increase as with the output gap measure, but now the magnitude of the increase is much larger and the HPIs for the 1955-1979 and 1982-2002 samples do not overlap. As a consequence of these changes, the standard deviation of the shocks to the first two equations shows a pattern which is the opposite of what we had in table 1: the standard deviation of the disturbance to the Euler equation slightly increases, while the one of the Phillips curve decreases. Surprisingly, the coefficient on inflation in the policy rule falls when we move from the pre-1979 to the post 1979 samples and the fall is significant. Taken at face value this implies that the policy rule has become less aggressive in responding to inflation since the beginning of the 1980s. While this may be due to the fact that inflation expectations were much less volatile in the 1980s, and therefore inflation stabilization may require a smaller coefficient, one should also recognize that differences across samples may reflect model misspecifications. The time profile of the standard deviation of the disturbance of the interest rate equation suggests that this is probably the case. Hence, despite being large, changes in the policy parameters account for little of the variations in interest rates.

Table 2 indicates that even with this policy rule the model has hard time to mimic the variability of the output gap and inflation, regardless of whether we use mean estimates or the percentiles where the actual values lies. As in the previous case, the variance of the output gap is underestimated and the one of inflation is typically overestimated but the magnitude of the discrepancy is larger with the former than with the latter. However, the estimates we obtain imply no volatility moderation. 


\begin{tabular}{|c|c|c|c|c|c|c|c|c|c|}
\hline & \multirow{3}{*}{\begin{tabular}{|c} 
Prior \\
Mean Std.
\end{tabular}} & \multicolumn{8}{|c|}{ Posterior } \\
\hline & & \multicolumn{2}{|c|}{ 1955-2002 } & \multicolumn{2}{|c|}{$1955-1979$} & \multicolumn{2}{|c|}{ 1979-2002 } & \multicolumn{2}{|c|}{ 1982-2002 } \\
\hline & & Mean & HPI & Mean & HPI & Mean & HPI & Mean & HPI \\
\hline$\varphi$ & $2.0 \quad 0.35$ & 2.56 & {$[1.81,2.90]$} & 2.36 & {$[2.61,3.22]$} & 2.71 & {$[2.63,2.93]$} & 2.05 & {$[1.62,2.74]$} \\
\hline$\beta$ & 0.980 .01 & 0.98 & {$[0.97,0.98]$} & 0.97 & {$[0.97,0.99]$} & 0.98 & {$[0.98,0.98]$} & 0.98 & {$[0.97,0.99]$} \\
\hline$\kappa$ & $2.0 \quad 1.0$ & 3.27 & {$[2.79,3.67]$} & 2.93 & {$[2.28,3.17]$} & 3.97 & {$[3.88,4.22]$} & 3.48 & {$[3.18,3.66]$} \\
\hline$\phi_{r}$ & $0.8 \quad 0.25$ & 0.98 & {$[0.98,0.99]$} & 0.99 & {$[0.99,0.99]$} & 0.99 & {$[0.99,0.99]$} & 0.99 & {$[0.99,0.99]$} \\
\hline$\phi_{x}$ & $0.5 \quad 0.25$ & 0.21 & {$[0.06,0.28]$} & -0.01 & {$[0.08,0.81]$} & 0.32 & {$[0.29,0.39]$} & -0.01 & {$[-0.02,0.00]$} \\
\hline$\phi_{\pi}$ & $\begin{array}{ll}1.3 & 0.5 \\
\end{array}$ & 1.34 & {$[1.00,1.46]$} & 1.02 & {$[1.00,1.05]$} & 1.69 & {$[1.65,1.72]$} & 1.52 & {$[1.24,1.76]$} \\
\hline$\rho_{x}$ & $0.85 \quad 0.25$ & 0.93 & {$[0.93,0.95]$} & 0.95 & {$[0.94,0.95]$} & 0.91 & {$[0.90,0.91]$} & 0.91 & {$[0.89,0.92]$} \\
\hline$\rho_{p}$ & $0.85 \quad 0.25$ & 0.99 & {$[0.99,0.99]$} & 0.99 & {$[0.99,0.99]$} & 0.96 & {$[0.96,0.97]$} & 0.98 & {$[0.98,0.98]$} \\
\hline$\sigma_{1}^{2}$ & 0.010 .50 & 0.027 & {$[0.020,0.032$} & 0.012 & {$[0.012,0.013]$} & 0.027 & {$[0.022,0.030]$} & 0.012 & {$[0.012,0.014]$} \\
\hline$\sigma_{2}^{2}$ & 0.010 .50 & 0.028 & {$[0.018,0.052$} & 0.061 & {$[0.063,0.063]$} & 0.161 & {$[0.128,0.193]$} & 0.066 & {$[0.049,0.068]$} \\
\hline$\sigma_{3}^{2}$ & 0.010 .50 & 0.015 & {$[0.012,0.015$} & 0.026 & {$[0.024,0.031]$} & 0.046 & {$[0.041,0.055]$} & 0.035 & {$[0.032,0.043]$} \\
\hline$a_{12}$ & $0.0 \quad 0.25$ & -1.05 & {$[-1.59,-0.66]$} & 0.13 & {$[-0.37,0.03]$} & 0.07 & {$[0.03,0.07]$} & 0.10 & {$[0.02,0.07]$} \\
\hline$a_{13}$ & $0.0 \quad 0.25$ & 1.25 & {$[0.79,1.46]$} & 0.13 & {$[0.25,0.35]$} & 0.13 & {$[0.10,0.13]$} & 0.10 & {$[0.10,0.14]$} \\
\hline Accep & & 0.50 & & 0.25 & & 0.30 & & 0.43 & \\
\hline Convergence after & & 22.000 & draws & 21.000 & draws & 19.000 & draws & 19.000 & draws \\
\hline
\end{tabular}

Table 4: Prior and Posterior Moments, Forward rule

With a policy rule which reacts to expected changes in the output gap and inflation, results are roughly similar. $\varphi$ falls as we move from the earlier to the later part of the sample but changes are smaller and the HPI of different samples overlap. $\kappa$ increases over time making the Phillips curve trade-off flatter and changes are a-posteriori significant. $\phi_{\pi}$ increases in the later part of the sample and the increase is now significant a-posteriori. With this specification of the policy rule, the mean estimate is on the boundary of the stability region in the first subsample suggesting that the likelihood of the data may be very sensitive to the specification of the policy rule. Once again, changes in the parameters of the policy rule account for little of the variations in the nominal interest rates and the changes in the standard deviation of the disturbance seem to do largely the job of matching the time path of the variance of interest rates.

A specification with a forward rule appears to be better in matching inflation variability than the original one but worse in matching output variability (see table 2). Furthermore, while it can reproduce the fall in the variances of the two variables in the last two subsamples, the fall in inflation in the 1982-2002 sample is small relative to the one observed in the data.

Overall, these alternative policy rules produce results which are qualitatively similar to 
the ones obtained in the baseline case, as far as trends in crucial parameter estimates are concerned. However, they seem to face more important problems in matching either the level or the decline in the volatility of output and inflation over the subsamples.

\section{A comparison with the literature}

Our findings may seem puzzling relative to what it is currently available in the literature, except perhaps for Gordon (2005). Hence, it is worth spending some time to discuss in what way our results are different and what can account for them.

To start with, we would like to point out three facts. First, our structural estimation does find an increase in the inflation coefficient of the policy rule when moving from a sample including the 1970s to a sample which excludes them. What we show is that the variations are not statistically large relative to those in other parameters. Second, time variations in parameters other than the policy ones are often detected when the model is estimated using systemwide methods (see Ireland (2001), Boivin and Giannoni (2006), among others), but they are left undiscussed. Third, direct structural estimation typically leads to conclusions that are different from those obtained by estimating structural VARs with or without time varying coefficients - the former mainly finds changes in the parameters of the model; the latter mainly changes in the covariance matrix of shocks. In some cases, this is due to the fact that variations in the standard deviations of the shocks cannot be identified with the chosen objective function (see e.g. Boivin and Giannoni (2006)); in others to specification choices which impose particular structure on the estimated structural shocks. Our findings, which are obtained conditioning on a model, are consistent with the VAR evidence.

Relative to Clarida et. al (2000), who use single equation structural estimation, we take a system wide estimation approach and use Bayesian rather than classical techniques. While the second difference may be of minor importance since the priors are sufficiently noninformative over the ranges we choose, the first one is important. Single equation methods may produce a distorted view of the structural relationships when important endogeneities are present (see also Lubik and Schorfheide (2004)). In addition, since they do not take into account system wide relationships, nor do they use the cross equation restrictions present 
in the model, they are inefficient.

In comparison with Boivin and Giannoni (2006), who use a minimum distance estimator to obtain parameter estimates, our approach has the advantage of allowing a better identification of the structural relationships. Canova and Sala (2006) have shown that minimum distance estimators when used to back out parameters of a new Keynesian model from the responses to monetary shocks face severe identification problems - the objective function is very flat and ridges are present. This means that variations in the coefficients identified by this procedure could be points of equivalent height on this surface or could represent variations linked to variations in the other parameters. On the contrary, the likelihood function of the system is much more peaked and displays much easily disentangable relationships among the parameters (see also Linde' (2005)). Two additional reasons may explain the different findings. First, the authors adjusts the estimated specification in order to achieve the best possible fit - endowing the theoretical model with ad-hoc exogenous frictions and searching among the (forward) specifications of the policy rule the one which best fit the interest rate data - while we take a textbook specification and do no preliminary data mining exercises. Table 4 shows that it is possible to roughly reproduce the pattern of point estimates they obtain with a one-period forward looking rule and no ad-hoc frictions. However, Boivin and Giannoni neglect the fact that pretesting downsizes the standard errors of their estimates. Hence, changes which are a-posteriori insignificant, may look artificially significant. Second, while the counterfactual exercises of Boivin and Giannoni are subject to the Lucas' critique - agents behave as if there will never be a structural break and when the break occurs they learn immediately that they will never be any break in the future the exercises we conduct in section 5 are largely free of these problems. As a matter of fact, the majority of the counterfactual exercises performed in the literature suffer from various types of inconsistencies which makes results uninterpretable. For example, the practice of switching coefficients and variances across samples does not take into account the correlation structure of estimates and the fact that the parameters/variances estimates obtained in a sample may be in the tails of estimated distribution of parameters/variances estimates in another sample. 
Relative to Lubik and Schorfheide (2004), who also employ system wide methods and Bayesian estimation on a model similar to ours, two important differences need to be mentioned. First, the policy rule they estimate uses output growth. As shown in table 2, this choice has some consequences for the results but does not change the main features of the conclusions one reaches. The second difference is that they allow for indeterminacy (and sunspots) in the estimation, while we don't. Consequently, this work complements rather than substitutes theirs.

Finally, several papers have estimated structural VARs with or without time variations in the coefficients (see e.g. Cogley and Sargent (2001), (2005), Canova and Gambetti (2004), Sims and Zha (2006) among others). While most of them are concerned with estimates of the policy rules and of the monetary policy shock, some papers have tried to estimate sources of variations in systems which have similarities with the model in section 2 (see e.g. Gambetti et. al. (2005)). Our results help to explain some of their findings. For example, the large impact that supply shocks have in explaining the time profile of the volatility and persistence of output is consistent with the time profile of the estimates of the coefficient of relative risk aversion in the log-linearized Euler equation. Moreover, the fall in the standard deviation of the supply shocks over time they find is due, in part, to the change in the Phillips curve trade-off $\kappa$.

\section{What change explains the Great Moderation?}

The analysis so far has documented the presence of generalized parameter instabilities over the samples under consideration and shown that only variations in certain parameters are statistically significant. In this section we ask which of these changes contributes most to the changes in the variance of output and inflation documented in figure 1. In particular, suppose we repeat estimation over the 1983-2002 subsample, fixing some parameters to their 1955-1974 posterior mean estimates. Would the fit of the model change? Would the model reproduce the fall in the volatility of output and inflation observed in this sample?

When examining which feature of the model is responsible for the Great Moderation, one typically performs counterfactual exercises where parameters for different subsamples 
are switched and interesting statistics are recomputed under these alternative parameter values. As we have mentioned, while popular in the literature (see e.g. Stock and Watson (2002), Biovin and Giannoni (2006)), these exercises cannot credibly answer the question of interest. Our approach, which allows unrestricted parameters to be readjusted in the estimation, can provide a more reasonable scenario to evaluate the economic consequences of parameter changes.

Table 5 reports estimates of the variance of the output gap and inflation obtained in the unrestricted specification and in three restricted specifications where, in turn, the parameters describing the private sector behavior $(\varphi, \kappa)$, the policy rule $\left(\phi_{x}, \phi_{r}, \phi_{\pi}\right)$, and the standard deviations of the shocks $\left(\sigma_{1}, \sigma_{2}, \sigma_{3}\right)$ are constrained to have a prior mean equal to the posterior mean of the 1955-1974 subsample and a very small variance (0.0001). Table 5 also reports the posterior probability of each model and the risk of matching the variance of output and inflation of the unrestricted model with each restricted specification.

Posterior probabilities are computed using the prior probability of each restricted specification (set to one-third) and their marginal likelihood. The marginal likelihood is a synthetic measure of fit, comparable to $\bar{R}^{2}$ in linear models: a higher marginal likelihood obtains if a model fits the data better, given a common prior, or if the prior of one model is closer to the likelihood, given a common likelihood. Since the experiments we conduct involve changing both the likelihood and the prior of the parameters, the marginal likelihood is altered through both channels. We compute marginal likelihoods using a modified harmonic mean estimator and 10 chains of parameter draws (see e.g. Geweke (1998)).

The risk measure is computed by comparing the volatilities of output and inflation produced by each restricted specification to the ones of the unrestricted specification under an absolute loss function, equally weighting the two volatilities by the posterior probability of each restricted specification. This type of measure, popularized in Schorfheide (2000), is useful to compare models which are likely to be misspecified and therefore may have very low posterior probability. We also computed a risk measure using a quadratic loss function or a loss which asymmetrically weights only positive deviations from the volatilities of the basic specification. The results we present are robust with respect to these choices. To 
interpret the risk measure note that if time variations in one set of parameters are relatively unimportant (important), the posterior probability of the restricted specification will be high (low) and the risk relatively high (low).

\begin{tabular}{|l|c|c|c|c|}
\hline \hline & Unrestricted & Restricting $\varphi, \kappa$ & Restricting $\psi_{x}, \psi_{r} \phi_{\pi}$ & Restricting $\sigma_{j}^{2}, j=1,2,3$ \\
\hline $\operatorname{Var}^{d}(\mathrm{Y})$ & 1.38 & & & \\
$\operatorname{Var}^{d}(\pi)$ & 1.06 & & & \\
$\operatorname{Var}(\mathrm{Y})$ & 0.80 & 0.05 & 2.98 & 4.57 \\
$\operatorname{Var}(\pi)$ & 16.39 & 1.27 & 47.04 & 14.97 \\
$\operatorname{Posterior}$ & & & & \\
$\operatorname{Probability}$ & & 0.999 & $3.0 \mathrm{e}-23$ & $2.7 \mathrm{e}-82$ \\
Risk & & 21.19 & $2.0 \mathrm{e}-21$ & $1.2 \mathrm{e}-81$ \\
\hline \hline
\end{tabular}

Table 5: Posterior Moments, Probabilities and Risk, restricted and unrestricted specifications, sample 1983-2002

Table 5 indicates that variations in the parameters of the private sector induce changes that go in the opposite directions of those we are interested here. In fact, if we keep them fixed at the posterior mean value estimated over the 1955-1974 sample, the fall in the variance of output and inflation implied by the model would have been much larger than in the unrestricted case. Hence, changes in $\varphi$ and $\kappa$ can not be the drivers of the "Great Moderation". Restricting the parameters of the policy rule to their 1955-1974 posterior mean values implies that the variability of output and inflation would have counterfactually increased rather than decreased over the 1982-2002 sample. Hence, the Great Moderation would not have occurred if policy parameters were invariant over the sample. Finally, restrictions on the volatility of the shocks have minor effects on the variance of inflation, but considerable effects on the variance of output. Consequently, the fall in the variances of output and inflation may have distinct causes: output volatility declines because of a combination of causes, among which the fall in the standard deviation of the shocks is the most important one. The fall in the variance of inflation, on the other hand, appears to be largely due to changes in the parameters of the policy rule.

How can one explain the extreme posterior probabilities of table 5? To start with, one should notice that Euler equation shocks are those with the lowest variability in all the samples. Therefore, given a shock of this type, changing the coefficient of relative 
risk aversion produces only small changes in the volatility of the output gap and, given the changes in the Phillips curve trade-off, this implies small variations in the volatility of inflation. On the contrary, small variations in the coefficients of the policy rule imply considerably different covariance matrices of the shocks and therefore large effects on the volatility of output and inflation. Finally, fixing the standard deviation of the shocks forces the parameters of the policy rule to change dramatically (for example, the output coefficient goes from 0.11 in the unrestricted specification to 0.99 when we fix the standard deviation of the shocks) and this has important consequences on the volatility of output produced by the model.

In sum, changes in the parameters of the policy rule and the variability of the shocks are crucial to understand the Great Moderation and, relatively speaking, posterior probabilities and risk measures suggest that time variations in the standard deviation of the shocks are the most important cause of the observed variations.

The results of this section should be seen as a warning against taking the results of statistical estimation at face value. Variations which are statistically large produce small economic consequences. On the contrary, small statistical variations, like those experienced in the parameters of the policy rule, may generate important economic implications because of the effects they have on the covariance structure of the shocks.

\section{Conclusions}

This paper recursively estimates a conventional small scale DSGE model using US postWWII data and Bayesian techniques. The model belongs to the class of New-Keynesian structures that have been extensively used in the current literature for welfare and policy analyses. Bayesian techniques are preferable to standard likelihood methods or to indirect inference (impulse response matching) exercises, especially for models like the one we con-

sider, which are clearly false and misspecified. We show that the model and the methodology are useful tools to understand the nature of the changes generating the so-called "Great Moderation".

We estimate the model a number of times using a different starting date, keeping the 
window size fixed, and analyze the role of changes in the private sector parameters, in the coefficients of the policy rule and in the covariance structure of the shocks. We find that changes over time in the parameters of the private sector are the largest and the most significant and tend to make the output gap be more elastic to changes in the real rate and inflation to be more reactive to marginal costs. Changes in the covariance structure of the shocks are also considerable while changes in the coefficients of the policy rule are small and a-posteriori insignificant.

Nevertheless, when we analyze which of these changes help to explain better the Great Moderation episode, we find that the changes in the parameters of the private sector alone cannot generate the observed fall in the variance of output and inflation while changes in the parameters of the policy rule and the covariance of the shocks can. We also show that the fall in variances of output and inflation appear to have different causes, suggesting that the quest for one common explanation to both facts is probably misplaced.

The results stand mid-way relative to those in the literature. As in structural VAR analyses, Canova and Gambetti (2004), Primiceri (2005) and Sims and Zha (2006), we find evidence that the shocks hitting the economy have considerably changed over time. Also, consistently with the analyses of McConnell and Perez Quiroz (2001), Gordon (2005) and Campbell and Herkovitz (2006), we detect statistical changes in the parameters of the private sector, but the changes matter very little to explain the Great Moderation episode. Finally, while policy parameters change little, they seem to matter quite a lot.

Our work has a number of limitations which we would like to spell out in detail. As we have mentioned, our analysis imposes the restriction that within each sample only a determinate equilibrium is present. This is relatively common in the literature (see e.g. Rabanal and Rubio Ramirez (2005) or Fernandez Villaverde and Rubio Ramirez (2007)) and, for the rolling analysis we perform, the restriction is probably less important that one would initially think. An obvious extension of what we have done here would be to allow for indeterminacies in every subsample and check whether rolling analysis would confirm or disproof our conclusions.

Second, our estimation approach, while convenient, imposes a form of irrationality on 
agents' behavior. In fact, the analysis implicitly assumes that agents have rational expectations within each sample where estimation is conducted but not over the entire sample they never take into account the fact that changes in the structural parameters may occur. To fully take this into account the techniques recently developed by Fernandez Villaverde and Rubio Ramirez (2007), which use higher order approximations to agents' decision rules and more complicated Monte Carlo techniques, need to be employed. This option, however, requires considerable computational time even in a model with only three equations.

Third, as we have argued in the introduction, the model is taken off-the-shelf and not optimized to fit the data in any sense. Therefore, there is always the possibility that misspecification, omitted variables or shocks drive the results. To fully understand the sources of the Great Moderation, one should probably employ a larger scale model which fits the data better than the simple specification we consider. Such an extension is relatively straightforward to undertake, but again requires considerable computational time.

Finally, while it is common to look at the US and only at output and inflation, there are obvious reasons to ask whether other variables display similar behavior and whether common explanations for the international patterns documented e.g. in Stock and Watson (2004) or Canova et. al. (2007), could be found. A cross-country perspective can be fundamental in understanding the source of variations because we know a lot about the policy changes and the dates at which they occurred in countries other than the US. We leave all these issues for future research. 


\section{References}

Arias, A., Hansen, G. and L. Ohanian (2006) Why have business cycle fluctuations become less volatile?, NBER Working Paper No. 12079.

Bernanke, B. and Mihov, I. (1998), "Measuring Monetary Policy", The Quarterly Journal of Economics, 113:869-902.

Blanchard, O.J. and Simon, J. (2000), ”The Long and Large Decline in U.S. Output Volatility", Brookings Papers on Economic Activity, 1, 135-147.

Boivin, J. and Giannoni, M. (2006), "Has Monetary Policy Become Less Powerful?", Review of Economics and Statistics, 88(3), 445-462.

Campbell, J. and Herkovitz, Z. (2006), "The Role of Collateralized Household Debt In Macroeconomic Stabilization", Federal Reserve Bank of Chicago, manuscript.

Canova, F. and Gambetti, L. (2004), "Structural changes in US economy: Is there a role for monetary policy?", available at www.econ.upf.edu/crei/people/canova.

Canova, F. and Gambetti, L. (2007), "Do inflation expectations matter? The Great Moderation revised", available at www.econ.upf.edu/crei/people/canova.

Canova, F., Gambetti, L., and E. Pappa (2007), " The structural dynamics of output growth and inflation: some international evidence", Economic Journal, 117, C167-C191.

Canova, F. and Sala, L. (2006), "Back to square one: identification issues in DSGE models", available at www.econ.upf.edu/crei/people/canova.

Cogley, T. and Sargent, T.J. (2001) "Evolving Post-World War II U.S. Inflation Dynamics", NBER Macroeconomic Annual, 16.

Cogley, T. and Sargent, T.J. (2005) "Drifts and Volatilities: Monetary Policies and Outcomes in the Post WWII U.S. ", Review of Economic Dynamics, 8. 262-302.

Clarida, R., J. Gali, and M. Gertler (1999), "The science of Monetary Policy: a Newkeynesian Perspective", Journal of Economic Literature, 37, 1661-1707.

Clarida, R., J. Gali and M. Gertler (2000), "Monetary Policy Rule and Macroeconomic Stability: Evidence and Some Theory", Quarterly Journal of Economics, CXV, 147-180.

Gambetti, L., E. Pappa and F. Canova (2005), "The structural dynamics of US output 
and inflation: what explains the changes? (with appendix)", CEPR working paper 5879, forthcoming, Journal of Money, Banking and Credit.

Fernandez Villaverde, J. and Rubio Ramirez, J. (2007) How structural are structural parameters?, forthcoming, NBER Macroeconomic Annual.

Geweke, J. (1998) "Using Simulation Methods for Bayesian Econometric Models: Inference, Development and Communication", Federal Reserve Bank of Minneapolis, Staff Report 249.

Gordon R. (2005), "What explain the decline in US business cycle volatility?", NBER working paper 11777 .

Hanson, M. (2006), "Varying Monetary Policy Regimes: a Vector Autoregressive Investigation", Journal of Business and Economics, 58, (5-6) 407-427.

Ireland, P. (1999) "Does the Time Consistent Problem Explain the Behavior of Inflation in the United States", Journal of Monetary Economics, 44, 279-292.

Ireland, P. (2001) "Sticky Price models of the Business Cycle: Specification and Stability" Journal of Monetary Economics, 47, 3-18.

Ireland, P. (2004) "Technology shocks in the New Keynesian Model", Review of Economics and Statistics, 86, 923-936.

Leeper, E. and Zha, T. (2003) "Modest Policy Interventions", Journal of Monetary Economics, 50, 1673-1700.

Linde', J. (2005), "Estimating New Keynesian Phillips curve: A Full Information Maximum Likelihood", Journal of Monetary Economics, 52, 1135-1149.

Lubik, T. and Schorfheide, F, (2004) "Testing for Indeterminacy: with an application to US monetary policy, American Economic Review, 94, 190-217.

McConnell, M. and Perez Quiroz, G. (2000) "Output fluctuations in the US: what has changed since the early 1980s?", American Economic Review, 90, 1464-1476.

Orphanides, A. (2004), "Monetary Policy Rules, Macroeconomic Stability, and Inflation: A View from the Trenches", Journal of Money, Banking and Credit, 36, 151-175.

Primiceri, G. (2005), "Time Varying Structural VAR and Monetary Policy, Review of Economic Studies, 72, 453-472. 
Pivetta, F. and Reis, R.(2007) "The Persistence of Inflation in the US", Journal of Economic Dynamics and Control, 31, 1326-1358.

Rabanal, P. and Rubio Ramirez, J. (2005), "Comparing New Keynesian Models of the Business Cycle: A Bayesian Approach ", Journal of Monetary Economics, 52, 1150-1169.

Smets, F. and Wouters, R. (2003), " An estimated DSGE model for the Euro area", Journal of the European Economic Association, 1, 1123-1175.

Smets, F. and Wouters, R. (2005), "Shocks and Frictions in US Business Cycles: a Bayesian DSGE approach", Journal of Applied Econometrics, 20, 161-183.

Sargent, T. (1999) The Conquest of American Inflation, Princeton University Press, Princeton, N.J.

Sims, C.A. and Zha, T. (2006), "Were there Regime Switches in US Monetary Policy?", American Economic Review, 96, 54-81.

Stock, J. and Watson, M. (2002) "Has the business cycle changed and Why", NBER Macroeconomic annual, Cambridge, MIT Press, 17, 159-218.

Taylor, J. (1998), "Monetary Policy Guidelines for Unemployment and Inflation Stability" in R. Solow and J. Taylor (eds.) Inflation, Unemployment and Monetary Policy, Cambridge, MA. 